\title{
Clinical Features and Outcomes of Streptococcus pneumoniae Meningitis in Children: A Retrospective Analysis of 26 Cases in China
}

\author{
Wenhui Wang ${ }^{1}$ Hong Han ${ }^{1}$ Lijun Du ${ }^{1}$ Zhaoyang $\mathrm{Li}^{1} \quad$ Yunhong Wu${ }^{1}$ \\ ${ }^{1}$ Department of Neurology, Children's Hospital of Shanxi, Taiyuan, \\ Shanxi, China \\ Address for correspondence Wenhui Wang, MS, Department of \\ Neurology, Children's Hospital of Shanxi, No. 13 Xinmin North Street, \\ Neuropediatrics 2022;53:32-38. \\ Xinghualing, Taiyuan, Shanxi 030013, China \\ (e-mail:wwhaeu@163.com).
}

\begin{abstract}
Background Streptococcus pneumoniae is an important cause of pediatric meningitis. Objective The aim of this study was to analyze the clinical features and outcomes of children with pneumococcal meningitis at our hospital in China, so as to provide basis for improving the clinical treatment effect.

Methods This retrospective analysis included patients aged $<16$ years treated for pneumococcal meningitis at the Department of Neurology, Children's Hospital of Shanxi (January 2014-February 2016). Clinical data were extracted from the medical records. Patients were followed up for 6 months after discharge.

Results The analysis included 26 children aged 2 months to 13 years, with $17(65.4 \%)$ aged $<3$ years. Presenting symptoms included fever (100\%), lethargy (100\%), impaired consciousness (88.5\%), neck stiffness (69.2\%), seizures (53.8\%), and headache (50.0\%). All patients had positive cerebrospinal fluid (CSF) cultures. The final treatment was vancomycin combined with a third-generation cephalosporin or other antibiotics in 25 patients. Eleven patients (42.3\%) were recovered, $3(11.5 \%)$ had neurological sequelae, and $12(46.2 \%)$ died. Impaired consciousness $(p=0.035)$, cerebral hernia $(p=0.037)$, respiratory failure $(p=0.004)$, heart

Keywords

- Streptococcus pneumoniae

- meningitis

- children

- infants

- neurological complications failure $(p=0.044)$, septic shock ( $p=0.037)$, low CSF white blood cell count $(p=0.036)$, high CSF protein levels ( $p=0.028)$, low white blood cell count $(p=0.036)$, and low blood neutrophil ratio $(p=0.016)$ are associated with a poor prognosis to pneumococcal meningitis.

Conclusion Pneumococcal meningitis is associated with a poor prognosis in many children. Poor prognosis might be related to early ineffective antibiotic therapy, a combination of systemic failure, neurological problems, and changed inflammatory response. It is important to rapid initiation of appropriate antibiotic therapy if meningitis is suspected.
\end{abstract}

\section{Introduction}

Bacterial meningitis in children and infants is associated with substantial morbidity and mortality. ${ }^{1}$ Although the incidence of bacterial meningitis has declined in Western countries following the introduction of vaccines against Haemophilus influenzae type b, Streptococcus pneumoniae, and Neisseria meningitidis, the incidence rate is much higher received

March 13, 2020 accepted after revision

February 14, 2021

published online

October 13, 2021
DOI https://doi.org/ 10.1055/s-0041-1728655. ISSN 0174-304X.

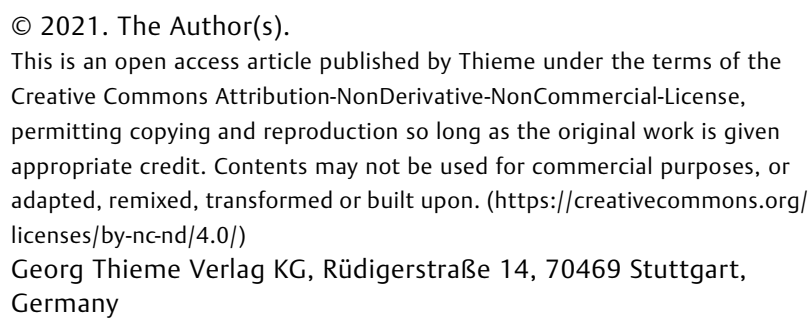


in developing regions of the world such as Africa. ${ }^{2}$ The recognized risk factors for pneumococcal meningitis include group B Streptococcus-positive mothers, immunodeficiency, absence of opsonic bactericidal antibody, basilar skull fracture, prematurity, low birth weight, young age, living in a dormitory, lack of immunization, ear/nose/throat infections, ventriculoperitoneal shunt, cochlear implants, and neurosurgery. ${ }^{1,3-6}$ Bacterial meningitis classically presents with fever, headache, signs of meningeal irritation, and altered level of consciousness, but these symptoms and signs may be hard to detect or absent in some cases, especially in neonates and infants. ${ }^{1}$ Neurological complications of bacterial meningitis include cerebral infarction, cerebral abscess, subdural empyema, cerebritis, and intracerebral bleeding, and these can lead to long-term sequelae such as focal neurological deficits, hearing loss, cognitive impairment, and epilepsy. ${ }^{7}$ Bacterial meningitis is potentially fatal, and the mortality rate is 10 to $15 \%$ in neonates. ${ }^{7}$

Streptococcus pneumoniae is a major cause of invasive diseases such as pneumonia, sepsis, and meningitis. ${ }^{8}$ Although the introduction of the pneumococcal conjugate vaccine has greatly reduced the global burden of invasive pneumococcal disease during the past two decades, it has been estimated that $S$. pneumoniae was responsible for 3.7 million cases of severe disease and more than 300,000 deaths in $2015 .{ }^{8}$ Pneumococcal meningitis is more frequently associated with worse outcomes than meningococcal meningitis, with a mortality rate of $35 \%$ and neuropsychological sequelae in $25 \%$ of those who survive. ${ }^{9}$ In China, $85 \%$ of pneumococcal diseases occur in children younger than 5 years, and antibiotic resistance is common in those with an invasive disease. ${ }^{10}$ Indeed, it is acknowledged that inappropriate use of antibiotics remains a problem in China and that increased access to pneumococcal vaccination is needed to reduce the incidence of pneumococcal meningitis in children and adults. ${ }^{11}$ During 2012 to 2015 , S. pneumoniae was the second most common pathogen isolated from the cerebrospinal fluid (CSF) of children with acute bacterial meningitis in Yunan Province and the most commonly isolated pathogen in infants aged $>3$ months, ${ }^{12}$ highlighting the importance of this bacterium as a cause of pediatric bacterial meningitis in China. However, data are limited regarding the clinical characteristics and outcomes of children with pneumococcal meningitis in China. ${ }^{13}$

The aim of this retrospective analysis was to analyze the clinical features and outcomes of children with pneumococcal meningitis treated at the Department of Neurology, Children's Hospital of Shanxi, China, between January 2014 and February 2016. It was anticipated that our findings might help clinicians identify patients at risk of poor outcomes such as neurological complications.

\section{Methods}

\section{Patients}

This was a retrospective analysis of all children (patients $<16$ years of age) who were treated for pneumococcal meningitis at the Department of Neurology, Children's Hospital of Shanxi (a 1,000-bed medical center) between January 2014 and February 2016. The inclusion criteria, based on the diagnostic criteria for pneumococcal meningitis, ${ }^{14}$ were as follows: (1) clinical manifestations of purulent meningitis in children; (2) the results of routine CSF tests, including biochemical investigations, were consistent with a diagnosis of purulent meningitis; and (3) S. pneumoniae was isolated on blood culture or CSF culture, and other pathogens were excluded. Patients were excluded from the study if the data required for the analysis were missing. This study was approved by the ethics committee of the Children's Hospital of Shanxi and the Women's Health Center of Shanxi, and the requirement for informed consent was waived.

\section{Collection of Clinical Data}

The following clinical information was extracted from the medical records: (1) age, sex, home location (urban or rural), vaccination status, age at onset, time from onset to admission, previous treatment received at a different hospital, hospital department to which first admitted, season of year in which the patient was admitted, and cause of death; (2) symptoms and signs, including fever, headache, vomiting, convulsions, and meningeal irritation; (3) results of blood tests on the day of admission (all within 8 days of disease onset), including routine blood tests, C-reactive protein (CRP) level, erythrocyte sedimentation rate (ESR), procalcitonin level, and blood culture; (4) results of CSF investigations during the first 3 days of admission (all within 11 days of disease onset), including routine CSF tests and CSF culture; (5) computed tomography (CT), magnetic resonance imaging (MRI), and electroencephalography (EEG) findings; (6) results of pathogen susceptibility tests; (7) presence of acute complications, including subdural effusion, hydrocephalus, and other neurological complications; and (8) clinical outcome at discharge, rated as 1 (death), 2 (persistent vegetative state), 3 (severe disability), 4 (moderate disability), or 5 (good recovery) using the Glasgow outcome scale (GOS). ${ }^{15}$ A GOS score of 5 was regarded as a good prognosis, and a GOS score of 0 to 4 was considered a poor prognosis. ${ }^{16}$

\section{Follow-up}

All patients were followed up by telephone at 6 months after discharge. The data collected at the follow-up included the frequency of any seizures, locomotor, cognitive, auditory, and visual function, and medication history.

\section{Statistical Analysis}

SPSS 17 (SPSS Inc., Chicago, Illinois, United States) was used to analyze the data. A descriptive statistical method was used. Continuous data are presented as median (range), and count data are presented as $n / N(\%)$. Continuous data were analyzed by the Kruskal-Wallis' test. Categorical data were analyzed by Fisher's exact test. A $p<0.05$ was considered statistically significant.

\section{Results}

Demographic Characteristics of the Study Participants and Clinical Manifestations of Pneumococcal Meningitis Among 525 children diagnosed with bacterial meningitis during the study period, 26 children (5.0\%) were confirmed 
34 Pneumococcal Meningitis in Children in China Wang et al.

Table 1 Demographic characteristics and clinical features for the 26 children with pneumococcal meningitis

\begin{tabular}{|c|c|c|c|}
\hline & Good prognosis $(n=11)$ & Poor prognosis $(n=15)$ & $p$-Value \\
\hline Age, y, median (range) & $1.4(0.2,13)$ & $1.8(0.3,13)$ & 0.979 \\
\hline Male, $n(\%)$ & $6(54.5)$ & $12(80.0)$ & 0.218 \\
\hline \multicolumn{4}{|l|}{ Source, $n(\%)$} \\
\hline City & $2(18.2)$ & $10(66.7)$ & \multirow[t]{2}{*}{0.658} \\
\hline Country & $9(81.8)$ & $5(33.3)$ & \\
\hline Time from onset to admission, $d$, median (range) & $2(1,8)$ & $3(1,7)$ & 0.671 \\
\hline Antibiotics within $3 \mathrm{~d}$ of onset, $n(\%)$ & $5(45.4)$ & $1(6.7)$ & 0.054 \\
\hline Dexamethasone, $n(\%)$ & $10(90.9)$ & $11(73.3)$ & 0.356 \\
\hline \multicolumn{4}{|l|}{ Clinical feature, $n(\%)$} \\
\hline Fever & $11(100)$ & $15(100)$ & $>0.99$ \\
\hline Lethargy & $11(100)$ & $15(100)$ & $>0.99$ \\
\hline Impaired consciousness & $8(72.7)$ & $15(100)$ & 0.035 \\
\hline Headache and vomiting & $6(54.5)$ & $7(46.7)$ & 0.697 \\
\hline Convulsions within $3 \mathrm{~d}$ of onset & $6(54.5)$ & $9(60)$ & 0.785 \\
\hline Hemiplegia & 0 & $3(20)$ & 0.122 \\
\hline Meningeal irritation signs & $8(72.7)$ & $9(60)$ & 0.509 \\
\hline Cerebral hernia & 0 & $5(33.3)$ & 0.037 \\
\hline DIC & 0 & $3(20)$ & 0.122 \\
\hline Respiratory failure & 0 & $8(53.3)$ & 0.004 \\
\hline Heart failure & $1(9.0)$ & $7(46.7)$ & 0.044 \\
\hline Renal failure & 0 & $1(6.7)$ & 0.392 \\
\hline Septic shock & 0 & $5(33.3)$ & 0.037 \\
\hline Bacteremia & $9(81.8)$ & $10(71.4)$ & 0.554 \\
\hline \multicolumn{4}{|l|}{ Cerebrospinal fluid tests, median (range) } \\
\hline White blood cell count & $990(20,4,700)$ & $148(1,9,600)$ & 0.036 \\
\hline Protein $(\mathrm{g} / \mathrm{L})$ & $2.7(1.6,4.7)$ & $8.7(1.0,22.7)$ & 0.028 \\
\hline Glucose (mmol/L) & $0.3(0.2,1.1)$ & $0.7(0.1,3.5)$ & 0.454 \\
\hline \multicolumn{4}{|l|}{ Blood tests, median (range) } \\
\hline White blood cell count $\left(10^{9} / \mathrm{L}\right)$ & $22.7(5.4,49.9)$ & $11.3(1.7,29.3)$ & 0.036 \\
\hline Neutrophil ratio (\%) & $85.4(58.0,92.2)$ & $68.8(19.0,91.6)$ & 0.016 \\
\hline Hemoglobin $(\mathrm{g} / \mathrm{L})$ & $116.0(70.0,121.0)$ & $115(91,176.8)$ & 0.516 \\
\hline Platelet count $\left(10^{9} / \mathrm{L}\right)$ & $246(71,609)$ & $170(22,547)$ & 0.287 \\
\hline C-reactive protein $(\mathrm{mg} / \mathrm{L})$ & $153.0(26.0,242.0)$ & $200.0(68.2,200.0)$ & 0.225 \\
\hline Procalcitonin (ng/mL) & $14.9(4.0,31.6)$ & $26.6(0.1,200.0)$ & 0.186 \\
\hline
\end{tabular}

Abbreviation: DIC, disseminated intravascular coagulation.

The significance of bold used in Table 1 , means $p<0.05$, statistical significance.

to have meningitis caused by S. pneumoniae. The demographic characteristics and clinical features of meningitis in these 26 cases (18 males, 69.2\%) are presented in - Table 1 and - Supplementary Table S1. The median age at onset was 1.5 years (range, 2 months-13 years), with 17 children (65.4\%) aged $<3$ years and 10 children (38.5\%) aged $<1$ year. None of the patients had been vaccinated against $S$. pneumoniae. The median duration from disease onset to admission was 2.5 days (range, $1-8$ days), and 12 patients had received antibiotics at other hospitals. The hospital's department to which the patient was first admitted was the neurology department in 12 cases, the pediatric intensive care unit (PICU) in 10 cases, cardiology department in 2 cases, and hematology department in 2 cases, and 9 patients were subsequently transferred to the PICU due to their critical clinical condition during treatment. The season of the year in which the patient was admitted was autumn (September-November) in 11 cases (42.3\%), winter (December-February) in 8 cases (30.8\%), spring (March-May) in 5 cases (19.2\%), and summer (June-August) in 2 cases (7.7\%). 
All patients presented with respiratory tract infection, fever, and lethargy. Headache and vomiting were present in 13 cases (50.0\%) and were more common in patients aged $\geq 1$ year than in those aged $<1$ year. Anterior fontanelle bulging was evident in all 10 patients aged $<1$ year. Impaired consciousness was a clinical manifestation in 23 cases (88.5\%). Seizures occurred in 14 patients (53.8\%), including 6 patients with seizures at disease onset; 6 patients had partial seizures, and 8 patients had generalized tonic-clonic seizures. Neck stiffness was present in 18 cases (69.2\%).

\section{Blood and CSF Tests}

The results of blood tests and CSF tests are shown in - Table 1 and - Supplementary Tables S2 and S3. Blood leukocyte count and platelet count were elevated in 14 cases (53.8\%) and 4 cases (15.4\%), respectively. All patients had high levels of procalcitonin and CRP, and 12 patients (46.2\%) had an elevated ESR $(>50 \mathrm{~mm} / \mathrm{h})$. CSF leukocyte count was elevated in 23 cases (88.5\%), and the neutrophil ratio ranged from 45 to $98 \%$. The CSF protein level exceeded $0.5 \mathrm{~g} / \mathrm{L}$ in all cases and was $>2.0 \mathrm{~g} / \mathrm{L}$ in 22 cases (84.6\%). The CSF glucose level was between 0.07 and 2.27 $\mathrm{mmol} / \mathrm{L}$ in 25 cases (96.2\%), and a slight decline in CSF chloride level was detected in 8 cases (30.8\%). CSF cultures were positive in all 26 cases, and 13 cases had a positive Gram stain in the CSF. Blood cultures were positive in 18 cases (69.2\%).

\section{Neuroimaging and EEG Findings}

Among 22 patients who received cranial MRI or CT, there were 11 cases $(50.0 \%)$ of intracranial multiple inflammatory lesions, 9 cases (34.6\%) of subdural effusion, 7 cases (31.8\%) of hydrocephalus, 4 cases (18.2\%) of diffuse cerebral edema, 4 cases $(18.2 \%)$ of brain atrophy, 2 cases $(9.1 \%)$ of focal cerebral hemorrhage, 2 cases (9.1\%) of cerebral softening, and 1 case (4.5\%) of dural venous sinus thrombosis (-Supplementary Table 54). In most cases, MRI suggests inflammatory or infectious lesions in various parts of the brain. Among the 26 patients, 16 (61.5\%) underwent cranial MRI. Among the 10 patients who did not receive MRI, 4 could not undergo any imaging at all because of a severe condition, and 6 only received skull CT examination. Among the 16 patients with MRI, 2 had normal results, 14 showed inflammatory changes (including 1 patient with local cortical hemorrhage and 1 with ischemic foci). The cerebral cortex was involved in all 14 patients. In addition, the ventricular system was involved in four patients, two patients had basal ganglia involvement, two patients had cerebellum involvement, two patients had diffuse cerebral atrophy, one patient had cerebral stem involvement, and one patient showed venous sinus and local venous thrombosis. Eight patients underwent an EEG examination: six cases showed slow background activity, and one case showed a large number of high-amplitude slow waves and sharp slow-wave bursts (-Supplementary Table S4).

\section{Acute Neurological Complications}

There were nine cases (34.6\%) of subdural effusion, seven cases (26.9\%) of hydrocephalus, six cases (23.1\%) of seizures, five cases $(19.2 \%)$ of cerebral hernia, four cases $(15.4 \%)$ of brain atrophy, three cases (11.5\%) of hemiplegia, two cases $(7.7 \%)$ of peripheral facial paralysis, and one case (3.8\%) each of subdural empyema, ependymitis, and dural venous sinus thrombosis.

\section{Nonneurological Complications}

There were nine cases (34.6\%) of bronchopneumonia, eight cases $(30.8 \%)$ of heart failure, eight cases $(30.8 \%)$ of respiratory failure, five cases (19.2\%) of septic shock, three cases (11.5\%) of diffuse intravascular coagulation, and one case (3.8\%) each of renal failure and suppurative otitis media.

\section{Treatment}

Twelve patients (46.2\%) were administered antibiotics prior to admission, including third-generation cephalosporins in eight cases (66.7\%), amoxicillin in two cases (16.7\%), erythromycin in one case $(8.3 \%)$, and cefuroxime in one case (8.3\%). All patients were treated with antibiotics on admission to our hospital. One patient (untreated before admission) was given cefoperazone monotherapy but died within 10 hours. The remaining 25 patients were initially treated with a third-generation cephalosporin, and the regimen was then switched to a vancomycincontaining regimen according to the results of drug sensitivity tests and therapeutic effects. The vancomycin-containing regimen included meropenem in 8 cases, cefotaxime in 9 cases, ceftriaxone in 1 case, cefoperazone/sulbactam in 17 cases, and ampicillin and cloxacillin in 2 cases. Susceptibility tests (-Table 2 ) revealed very high resistance rates to erythromycin (100\%) and clindamycin (95.8\%) as well as substantial resistance to penicillin (53.8\%), cefotaxime (34.6\%), and ceftriaxone (34.6\%). However, all strains were susceptible to vancomycin, meropenem, levofloxacin, and linezolid ( - Table $\mathbf{2}$ ). In addition to the rational use of antibiotics, albumin was coadministered in 16 patients, and large-dose gamma globulin was used in 12 cases. All children received mannitol infusion to reduce intracranial pressure as well as other symptomatic and supportive treatments. Steroids were used in 21 patients (dexamethasone $0.3-0.5 \mathrm{mg} / \mathrm{kg} / \mathrm{d}$, every 12 hours).

\section{Outcomes}

Univariable analyses were used to explore the factors associated with prognosis. They suggest that impaired consciousness $(p=0.035)$, cerebral hernia $(p=0.037)$, respiratory failure

Table 2 Results of CSF test for drug susceptibility tests

\begin{tabular}{|l|l|l|}
\hline Antibiotic drug & $\begin{array}{l}\text { Drug-resistant } \\
\text { strains/all cases }\end{array}$ & $\begin{array}{l}\text { Resistance } \\
\text { rate (\%) }\end{array}$ \\
\hline Clindamycin & $23 / 24$ & $95.8 \%$ \\
\hline Ceftriaxone & $9 / 26$ & $34.6 \%$ \\
\hline Erythromycin & $24 / 24$ & $100 \%$ \\
\hline Levofloxacin & $0 / 26$ & $0 \%$ \\
\hline Linezolid & $0 / 26$ & $0 \%$ \\
\hline Penicillin & $14 / 26$ & $53.8 \%$ \\
\hline Cefotaxime & $9 / 26$ & $34.6 \%$ \\
\hline Vancomycin & $0 / 26$ & $0 \%$ \\
\hline Meropenem & $0 / 2$ & $0 \%$ \\
\hline
\end{tabular}

Abbreviation: CSF, cerebrospinal fluid. 
( $p=0.004)$, heart failure $(p=0.044)$, septic shock $(p=0.037)$, low CSF white blood cell count $(p=0.036)$, high CSF protein levels $(p=0.028)$, low white blood cell count $(p=0.036)$, and low blood neutrophil ratio $(p=0.016)$ are associated with a poor prognosis to pneumococcal meningitis.

Seven of the patients treated with vancomycin recovered within 1 week $(\mathrm{GOS}=5)$. Further four patients showed marked improvement (conscious and without fever, but with some abnormal CSF parameters) and left the hospital voluntarily; at the 6-month follow-up, all four of these patients underwent CSF reexamination to verify whether the previous abnormalities were still present but had normal results for CSF tests and had no neurological sequelae ( $G O S=5$ ). Therefore, 11 of the 26 patients (42.3\%) had a good prognosis. Among the 15 patients (57.7\%) with a poor prognosis, 3 patients had neurological sequelae (hydrocephalus, hemiplegia, cognitive impairment, and epilepsy; GOS=3), and 12 patients died. The causes of death were multiple organ failure $(n=4)$, cerebral hernia $(n=3)$, and septic shock $(n=5)$.

\section{Discussion}

In the present study, almost two-thirds of the children were $<3$ years of age, and nearly $40 \%$ were aged $<1$ year, which is consistent with previous reports that the majority of children with pneumococcal meningitis were aged $<3$ years. ${ }^{13,17,18}$ This likely reflects the immaturity of the immune system in infants since the passive immunity passed on by the mother is lost within the first few months after birth. The ratio of males to females in our cohort of patients was $2.25: 1$, in broad agreement with previous findings indicating that pneumococcal meningitis was roughly twice as common in boys than girls. $^{12,17}$ Furthermore, $73.1 \%$ of the cases in our study occurred during the autumn or winter season, which concurs with previous investigations. ${ }^{12}$ The apparent association between pneumococcal meningitis and winter season may, in part, be due to higher levels of air pollution at this time of year, which has also been suggested to increase the risk of invasive pneumococcal disease. ${ }^{19}$ Pneumococcal infections per se show increased prevalence rates during winter which may be another important cause. A variety of other factors have also been suggested to be related to bacterial meningitis in children, including poor weight gain (malnutrition), parental smoking, high house crowdedness, cochlear implants, and underlying medical conditions such as sinusitis, otitis, HIV infection, immunodeficiency, and CSF leak. ${ }^{20}$

In this study, the presenting symptoms included respiratory tract infection (100\%), fever (100\%), lethargy (100\%), impaired consciousness (88.5\%), neck stiffness (69.2\%), seizures $(53.8 \%)$, headache $(50.0 \%)$, and vomiting $(50.0 \%)$, while anterior fontanelle bulging was evident in all patients aged $<1$ year. Our findings are broadly consistent with a previous report of Chinese children (12) and another from Hungary. ${ }^{21}$ In the present study, nine patients (34.6\%) had pneumonia, five patients (19.2\%) had sepsis, and one patient (3.8\%) had suppurative otitis media, suggesting that pneumococcal meningitis often occurs following other systemic infections such as pneumonia and sepsis. The low incidence of pneu- monia was probably related to the low positive rate of culture. All patients included in this study had elevated levels of CSF protein, serum procalcitonin, and serum CRP, and most had elevated CSF leukocyte count and low CSF glucose level, as supported by a previous study. ${ }^{22}$ Nevertheless, it is important to highlight that $11.5 \%$ of the children had normal CSF cell counts, suggesting that CSF cell count should not be performed alone since it can be normal in some children; CSF protein and glucose levels should also be assessed. Notably, less than half the patients had a high white blood cell count in peripheral blood, indicating that this may be a less reliable indicator of pneumococcal meningitis. On the one hand, low CSF and blood leukocyte counts do not rule out the diagnosis of pneumococcal meningitis. On the other hand, both factors are also associated with poor prognosis which might point to a problem with the immune response or consumption of leukocytes in the course of septicemia.

An important finding of the present study was that neurological complications were common in children with pneumococcal meningitis and included subdural effusion (34.6\%), hydrocephalus (26.9\%), epilepsy (23.1\%), cerebral hernia (19.2\%), brain atrophy (15.4\%), hemiplegia (11.5\%), peripheral facial paralysis (7.7\%), subdural empyema (3.8\%), ependymitis (3.8\%), and dural venous sinus thrombosis (3.8\%). Previous studies also reported high rates in such patients. ${ }^{9,18,21,23,24}$ The mortality rate among the children in our study was $46.2 \%$, somewhat higher than the rates reported previously, ${ }^{9,18,21,23,24}$ and 7 of these 12 patients (58.3\%) were $<2$ years of age. The reasons underlying the higher mortality rate in our study are not known, although one contributing factor may be the high proportion of patients $(46.2 \%)$ treated with antibiotics at other hospitals prior to admission to our institution: ineffective therapy at other hospitals may have delayed the initiation of appropriate antibiotic therapy and thus resulted in poorer outcomes. Poor prognosis might also be related to a combination of systemic failure, neurological problems, and changed inflammatory response. In a previous study, the factors associated with a poor prognosis included younger age, lengthy fever, low CSF cell count, very low CSF glucose, low neutrophil count, resistance to penicillin, low GOS, organ failure, and early admission to intensive care unit. ${ }^{25}$ Systemic factors might be important regarding mortality, but neurological problems might be more important regarding the outcomes in survivors. This will have to be examined in future studies with larger sample sizes. Nevertheless, our findings and previous studies emphasize the need to instigate appropriate treatment as early as possible to minimize the risks of poor outcomes.

The vancomycin/rifampin combination is an alternative for patients with allergy to penicillin or cephalosporins, and other drugs such as meropenem or chloramphenicol can be considered. The antibiotic regimen is then adjusted once the CSF culture and sensitivity results are known. The recommended empiric regimen for children aged $>1$ month is ceftriaxone or cefotaxime plus vancomycin or rifampicin. ${ }^{26}$ In this study, 25 patients were treated empirically with 
vancomycin after admission; in 24 patients, vancomycin was combined with a third-generation cephalosporin, as recommended, and in 7 patients, vancomycin was subsequently administered with meropenem because of poor initial efficacy, critical clinical condition, and/or the results of susceptibility tests. One patient was not given vancomycin because of death 10 hours after admission. The resistance of $S$. pneumoniae to cephalosporins showed an increasing trend in China, and the highest sensitivities were observed with levofloxacin, moxifloxacin, and vancomycin. ${ }^{10,27}$ Ceftaroline might also be a good choice. ${ }^{28}$ This cephalosporin resistance of S. pneumoniae is not exclusive in China. ${ }^{29-31}$ In the present study, 14 patients $(53.8 \%)$ had penicillin-resistant strains who were also resistant to erythromycin, but all strains were sensitive to vancomycin. Previous studies have reported penicillin resistance rates of 31 to $89 \%$, ceftriaxone resistance rates of 14 to $52 \%$, and erythromycin resistance rates approaching $100 \%$, but full susceptibility to vancomycin. ${ }^{10,12,13}$ On account of the high mortality and morbidity associated with pneumococcal meningitis, the high level of penicillin resistance in our pneumococcal meningeal isolates leads us to believe that it would be prudent to add vancomycin to ceftriaxone for initial treatment of acute bacterial meningitis till culture reports become available, after which antibiotics can be administered based on results of susceptibility testing. Local antibiotic prescribing patterns affect the local resistance patterns, ${ }^{32}$ highlighting the need for susceptibility testing in each case.

In this study, the mortality rate of pneumococcal meningitis was $46.2 \%$ (12/26). Reported mortality rates were $20.3 \%$ $(65 / 321)$ in New York (United States), ${ }^{33} 20.1 \%$ (41/204) in Chicago (United States), ${ }^{34} 12.7 \%(107 / 841)$ in the Netherlands, ${ }^{8} 13.1 \%(704 / 5,374)$ in Denmark, ${ }^{35} 1.1 \%$ (3/264) in Germany, ${ }^{36}$ 5.5\% (3/55) in 2003 to 2015 versus $24.1 \%(21 / 87)$ in 1984 to 2002 in Germany, ${ }^{37} 13.7 \%$ (42/306) in Turkey, ${ }^{38}$ and $21.4 \%$ (6/28) in Gambia. ${ }^{39}$ A meta-analysis indicated that the mortality rate varied according to the serotype of $S$. pneumoniae, ${ }^{40}$ but the present study did not examine the exact serotype.

This study has some limitations. This was a retrospective analysis, so the results may be prone to information bias and selection bias. The generalizability of the findings is not known because this was a single-center study with a small sample size. Streptococcus pneumoniae serotyping was not performed, so the causative serotypes and their possible associations with outcomes were not evaluated. Due to the small sample size, multivariable analysis of factors independently associated with poor outcomes could not be performed.

\section{Conclusion}

In conclusion, pneumococcal meningitis was associated with neurological sequelae or death in a majority of children in China. Poor prognosis might be related to early ineffective antibiotic therapy, a combination of systemic failure, neurological problems, and changed inflammatory response. Fur- thermore, the resistance rates of $S$. pneumoniae to penicillin were high, and this emphasizes the need for rapid initiation of using appropriate empiric antibiotic therapy such as combination therapy with vancomycin added to cefotaxime or ceftriaxone if meningitis is suspected and adjustment of the antibiotic regimen once the results of susceptibility tests are known.

\section{Funding \\ None.}

Conflict of Interest

None declared.

\section{Acknowledgments}

The authors would like to thank the patients and their families who participated in this study. The authors would also like to thank Xiaoli Sun, Hao Sun, Lifang Jia, Chunhua Li, Yanmei Wang, and Linxia Zhang (physicians at the Children's Hospital of Shanxi) for their help.

\section{References}

1 Kim KS. Acute bacterial meningitis in infants and children. Lancet Infect Dis 2010;10(01):32-42

2 Brouwer MC, van de Beek D. Epidemiology of community-acquired bacterial meningitis. Curr Opin Infect Dis 2018;31(01):78-84

3 Tunkel AR, Hartman BJ, Kaplan SL, et al. Practice guidelines for the management of bacterial meningitis. Clin Infect Dis 2004;39(09): $1267-1284$

4 Le Saux NCanadian Paediatric Society, Infectious Diseases and Immunization Committee. Guidelines for the management of suspected and confirmed bacterial meningitis in Canadian children older than one month of age. Paediatr Child Health 2014;19 (03):141-152

5 National Institute for Health and Care Excellence (NICE) Meningitis (bacterial) and meningococcal septicaemia in under 16s: recognition, diagnosis and management. CG102 Accessed July 9, 2020 at: https://www-nice-org-uk.acces.bibl.ulaval.ca/guidance/ cg102/resources/meningitis-bacterial-and-meningococcal-septicaemia-in-under-16s-recognition-diagnosis-and-managementpdf-35109325611205

6 Mount HR, Boyle SD. Aseptic and bacterial meningitis: evaluation, treatment, and prevention. Am Fam Physician 2017;96(05): 314-322

7 Baud O, Aujard Y. Neonatal bacterial meningitis. Handb Clin Neurol 2013;112:1109-1113

8 Jansen AG, Rodenburg GD, van der Ende A, et al. Invasive pneumococcal disease among adults: associations among serotypes, disease characteristics, and outcome. Clin Infect Dis 2009;49(02):e23-e29

9 Ramakrishnan M, Ulland AJ, Steinhardt LC, Moïsi JC, Were F, Levine OS. Sequelae due to bacterial meningitis among African children: a systematic literature review. BMC Med 2009;7:47

10 Wang $\mathrm{CY}$, Chen $\mathrm{YH}$, Fang $\mathrm{C}$, et al. Antibiotic resistance profiles and multidrug resistance patterns of Streptococcus pneumoniae in pediatrics: a multicenter retrospective study in mainland China. Medicine (Baltimore) 2019;98(24):e15942

11 Jiang $\mathrm{H}$, Huai $\mathrm{Y}$, Chen $\mathrm{H}$, et al. Invasive Streptococcus pneumoniae infection among hospitalized patients in Jingzhou city, China, 2010-2012. PLoS One 2018;13(08):e0201312

12 Jiang H, Su M, Kui L, et al. Prevalence and antibiotic resistance profiles of cerebrospinal fluid pathogens in children with acute bacterial meningitis in Yunnan province, China, 2012-2015. PLoS One 2017;12(06):e0180161 
13 Su M, Chang L, Zhou W, Mu LY, Kuang LH. Clinical characteristics of children with meningitis caused by Streptococcus pneumoniae and antibiotic resistance of Streptococcus pneumoniae strains. Zhongguo Dang Dai Er Ke Za Zhi 2015;17(07):706-709

14 WuXR, LQ. Basic and Clinical Aspects of Pediatric Neurological Diseases. 2nd ed. Beijing: People's Health Publishing House; 2009:472-477

15 Jennett B, Bond M. Assessment of outcome after severe brain damage. Lancet 1975;1(7905):480-484

$16 \mathrm{Hsu}$ MH, Hsu JF, Kuo HC, et al. Neurological complications in young infants with acute bacterial meningitis. Front Neurol 2018;9:903

17 Rumlarová Š, Kosina P, Kračmarová R, Plíšek S, Rejtar P. Pneumococcal meningitis in children. Klin Mikrobiol Infekc Lek 2013;19 (04):128-131

18 Hua F, Shang S, Yang Y-W, et al. TRIB3 interacts with $\beta$-catenin and TCF4 to increase stem cell features of colorectal cancer stem cells and tumorigenesis. Gastroenterology 2019;156(03):708-721.e15

19 Kim PE, Musher DM, Glezen WP, Rodriguez-Barradas MC, Nahm WK, Wright CE. Association of invasive pneumococcal disease with season, atmospheric conditions, air pollution, and the isolation of respiratory viruses. Clin Infect Dis 1996;22(01):100-106

20 Hénaff F, Levy C, Cohen R, et al; French Group of Pediatric Infectious Diseases (GPIP) Risk factors in children older than 5 years with pneumococcal meningitis: data from a national network. Pediatr Infect Dis J 2017;36(05):457-461

21 Ivády B, Liptai Z, Ujhelyi E, Balázs G. Pneumococcal meningitis in children-9 1/2-year-experience at Szent László Hospital, Budapest, Hungary. Ideggyogy Sz 2008;61(11-12):385-390

22 Tacon CL, Flower O. Diagnosis and management of bacterial meningitis in the paediatric population: a review. Emerg Med Int 2012;2012:320309

23 Berberian G, Pérez MG, Epelbaum C, Ceinos MdelC, Lopardo H, Rosanova MT. Pneumococcal meningitis: a 12 year experience in a children's hospital prior to the universal immunization with a conjugate vaccine. Arch Argent Pediatr 2014;112(04): 332-336

24 Lucey JM, Gavin P, Cafferkey M, Butler KM. Pneumococcal meningitis: clinical outcomes in a pre-vaccine era at a Dublin paediatric hospital, 1999-2007. Ir J Med Sci 2011;180(01):47-50

25 Jordan I, Calzada Y, Monfort L, et al. Clinical, biochemical and microbiological factors associated with the prognosis of pneumococcal meningitis in children. Enferm Infecc Microbiol Clin 2016;34(02):101-107

26 van de Beek D, Cabellos C, Dzupova O, et al; ESCMID Study Group for Infections of the Brain (ESGIB) ESCMID guideline: diagnosis and treatment of acute bacterial meningitis. Clin Microbiol Infect 2016;22(Suppl 3):S37-S62
27 Zhao C, Li Z, Zhang F, et al. Serotype distribution and antibiotic resistance of Streptococcus pneumoniae isolates from 17 Chinese cities from 2011 to 2016. BMC Infect Dis 2017;17(01):804

28 Kosowska-Shick K, McGhee PL, Appelbaum PC. Affinity of ceftaroline and other beta-lactams for penicillin-binding proteins from Staphylococcus aureus and Streptococcus pneumoniae. Antimicrob Agents Chemother 2010;54(05):1670-1677

29 Klugman KP, Feldman C. Penicillin- and cephalosporin-resistant Streptococcus pneumoniae. Emerging treatment for an emerging problem. Drugs 1999;58(01):1-4

30 Kim L, McGee L, Tomczyk S, Beall B. Biological and epidemiological features of antibiotic-resistant Streptococcus pneumoniae in pre- and post-conjugate vaccine eras: a United States perspective. Clin Microbiol Rev 2016;29(03):525-552

31 Chenoweth CE, Saint S, Martinez F, Lynch JP III, Fendrick AM. Antimicrobial resistance in Streptococcus pneumoniae: implications for patients with community-acquired pneumonia. Mayo Clin Proc 2000;75(11):1161-1168

32 Hicks LA, Chien YW, Taylor TH Jr, Haber M, Klugman KPActive Bacterial Core Surveillance (ABCs) Team. Outpatient antibiotic prescribing and nonsusceptible Streptococcus pneumoniae in the United States, 1996-2003. Clin Infect Dis 2011;53(07):631-639

33 Austrian R, Gold J. Pneumococcal bacteremia with especial reference to bacteremic pneumococcal pneumonia. Ann Intern Med 1964;60:759-776

34 Mufson MA, Kruss DM, Wasil RE, Metzger WI. Capsular types and outcome of bacteremic pneumococcal disease in the antibiotic era. Arch Intern Med 1974; 134(03):505-510

35 Harboe ZB, Thomsen RW, Riis A, et al. Pneumococcal serotypes and mortality following invasive pneumococcal disease: a population-based cohort study. PLoS Med 2009;6(05):e1000081

36 Rückinger S, von Kries R, Siedler A, van der Linden M. Association of serotype of Streptococcus pneumoniae with risk of severe and fatal outcome. Pediatr Infect Dis J 2009;28(02):118-122

37 Buchholz G, Koedel U, Pfister HW, Kastenbauer S, Klein M. Dramatic reduction of mortality in pneumococcal meningitis. Crit Care 2016;20(01):312

38 Erdem H, Elaldi N, Öztoprak N, et al. Mortality indicators in pneumococcal meningitis: therapeutic implications. Int J Infect Dis 2014;19:13-19

39 Antonio M, Dada-Adegbola H, Biney E, et al. Molecular epidemiology of pneumococci obtained from Gambian children aged 2-29 months with invasive pneumococcal disease during a trial of a 9valent pneumococcal conjugate vaccine. BMC Infect Dis 2008;8:81

40 Weinberger DM, Harboe ZB, Sanders EA, et al. Association of serotype with risk of death due to pneumococcal pneumonia: a meta-analysis. Clin Infect Dis 2010;51(06):692-699 